\title{
Code Smoothing for BOC Ambiguity Mitigation
}

\author{
Moisés Navarro Gallardo ${ }^{(1,2)}$, Gonzalo Seco Granados ${ }^{(1)}$ Gustavo López Risueño $^{(2)}$, and Massimo Crisci ${ }^{(2)}$ \\ (1) Universitat Autònoma de Barcelona (UAB), Spain \\ (2) European Space Agency (ESA), The Netherlands \\ e-mails: \{moises.navarro;gustavo.lopez;massimo.crisci\}@esa.int, \{gonzalo.seco\}@uab.cat
}

\begin{abstract}
The most recent generation of Global Navigation Satellite Systems (GNSS) are implementing Binary Offset Carrier (BOC) modulation. These signals are expected to provide not only better precision in the estimation of the signal's delay and phase but also more robustness to multipath effects. The advantage of BOC signals is that the main lobe of the correlation is very narrow, but on the other hand they present side lobes. For high-order signals, the amplitude of the side lobes can be similar to the amplitude of the main one or even exceed it under specific scenarios. Some techniques to mitigate the code ambiguity exploit the fact that BOC signals can be understood as the sum of two BPSK signals. Even though these techniques achieve their objective, they lose the robustness against multipath and increase the tracking noise. This paper presents a new combination between the time delay estimated by these kind of techniques and the time delay estimated using the full BOC. The idea of the combination is the same as the carrier smoothing but instead of using the carrier measurement, two code measurements are combined. Since the delay introduced by the ionosphere is the same, or very close, using the Full-BOC and the two-BPSK techniques, as it will be shown in this paper, the smoothing time can be large values, compared with the common carrier smoothing time. Several simulations of the new code smoothing strategy for different scenarios are presented in this paper.
\end{abstract}

Index Terms-Ambiguity, BOC, BOCcos, side lobes.

\section{INTRODUCTION}

The BOC (Binary Offset Carrier) signals have been chosen for the new generation of GNSS (Global Navigation Satellite System) as Galileo, the upgrade of the GPS system and the future Chinese (COMPASS/BEIDOU) and Indian (GAGAN) systems. These signals were expected to provide not only better precision in the estimation of the signals delay and phase but also more robustness to multipath effects (i.e. the effect whereby the transmitted signal reaches the receiver through different paths that experience different delays and attenuations). These contributions are combined with the direct signal causing an error in the estimation of the position.

The ambiguity issue with the BOC signals is a well-known problem in the GNSS community: confusing a side lobe with the main lobe (synchronization error) and hence producing an error in the computation of the position. This issue becomes more challenging as the order of the BOC increases. For instance, the autocorrelation of $\operatorname{BOC}(15,2.5)$ signal has the side lobes only at 9.7 meters of the main one and their value is around 0.89 (normalised assuming that the maximum is equal to one). Several methods with the aim of mitigating the ambiguity have been presented in the literature. One kind of them are called BPSK-like. They are based on seeing the BOC signals as the sum of two BPSK signals [1] [2]. They can be filtered by one filter that has enough bandwidth for both BPSK, or each BPSK can be filtered using different filters centered at the carrier frequency $+/$ - the sub-carrier frequency. In [3] the authors analyze the behavior of using only one BPSK signal instead of the combination of two. Another unambiguous technique that achieves the BPSK, or close, shape is based on taking out the sub-carrier component as it is usually done with the carrier [4]. Although these methods are able to mitigate the ambiguity issue, they lose all the multipath mitigation properties of the BOC signals. The Double Estimator (DE) [5] is based on the use of three loops: Phase Lock Loop (PLL) for the phase delay, Delay Lock Loop (DLL) for the code delay and a new one for the sub-carrier delay, the Sub-carrier Lock Loop (SLL). It means that, the code and sub-carrier delays could be different. Therefore, a 2D correlation are obtained. It combines both dimensions, i.e. the DLL and SLL, in order to achieve a unambiguous correlation. Although the BPSK-Like and the DE are completely different, one dimension of the correlation obtained with the DE has the same shape than the BPSK-like. The idea of the DE technique is to correct the SLL using the DLL. If the different between them is bigger than half sub-carrier cycle, the measurement jumps to the right peak. In [6] the author presents different ways to combine the two measures obtained with the DE technique.

In this paper a new idea in order to mitigate the ambiguity problem is presented. It is based on the use of two code delays: one that is ambiguous and another one that is unambiguous. The goal is to apply code smoothing: the same idea as in carrier smoothing but using two code measurements instead of one phase and one code measurements. Using the BPSK-like techniques an unambiguous but noisy delay is achieved. The delay estimated using the full BOC signal is ambiguous but less noisy. There is a big similitude between these two delays and the two delays that are used in carrier smoothing strategy: the code measurements are unambiguous but noised, whereas that the carrier measurements are less noisy but ambiguous. One of the carrier smoothing problems is that the carrier and the code are delayed differently by the ionosphere. Hence, a bias between both measurement is introduced and it is time variant. This effect limits the smoothing time. Since the BPSK and BOC delays are close to the same frequency, the ionosphere effect is practically the same for both code delays. 
This is shown in the following sections.

The remaining of this document is organized as follows. In Section II the fact that the BOC signals can be expressed as the sum of BPSK is presented. The Section III exposes the mathematical expressions of the smoothing strategy and its common version carrier smoothing. The new unambiguous technique based on the smoothing strategy is presented in Section IV. The ionosphere effects and its impact on the BPSK-like and BOC code delay are presented in Section V. The combination of the two BPSK measurements is shown in Section VI. The results and the conclusions are presented in Section VII and Section VIII.

\section{DuAl SidebAnd}

The fact that the BOC signals can be tracked as the sum of two BPSK is a well-known ambiguity mitigation method. Actually, it can be shown that the BOCcos pulses can be written as sum of infinite sinusoids using the Fourier series as

$$
p_{s c}(t)=\sum_{n=1}^{\infty} \frac{4(-1)^{(n+1)}}{\pi(2 n-1)} \cos \left(w_{0}(2 n-1) t\right) p(t),
$$

where $w_{o}=2 \pi / T_{o}, T_{o}$ is the inverse of the sub-carrier frequency, and $p(t)$ is a square pulse of duration equal to inverse the chip rate. The amplitude is decreasing as $n$ increases. Since the signal is filtered, the expression can be reduced to the first term, i.e. $n=1$, neglecting the higher terms. The Fig. 1 shows the spectrum of two BPSK centered at $+/-$ the sub-carrier frequency, the theoretical BOCcos and the Fourier series for $n=1$. The Bandwidth of the satellite has been set at $+/-19 \mathrm{MHz}$ as a brick wall filter. It should be noted that, inside the bandwidth, the three spectra are very similar to each other. Hence, the filtered BOCcos signal can be expressed, in terms of the spectrum as the sum of two BPSK.

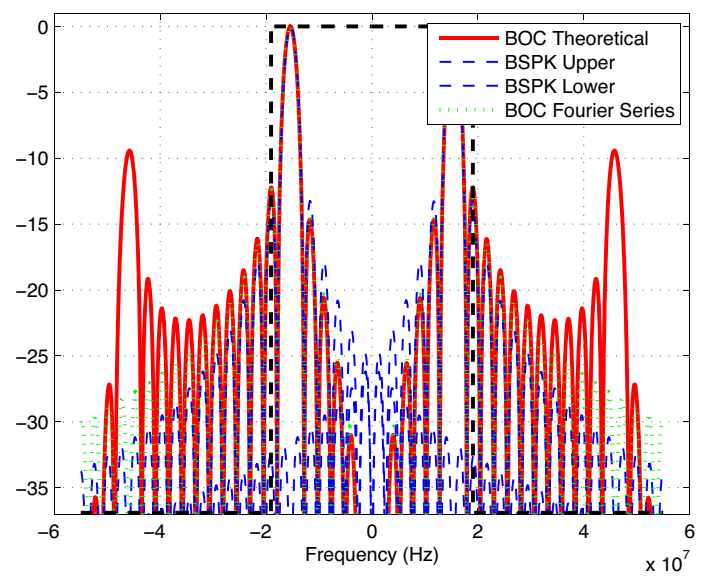

Fig. 1. BOCcos $(15,2.5)$ Spectrum

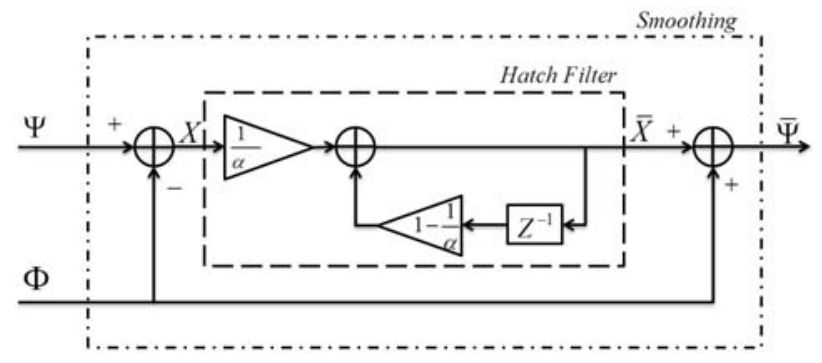

Fig. 2. Smoothing block diagram

\section{SMOOTHING STRATEGY}

\section{A. Hatch Filter}

The most common smoothing technique is based on the Hatch filter [7], [8]. The idea is to smooth an unbiased but noisy measurement using a biased but non-noisy measurement. For instance, the code is smoothed using the carrier measurements. Fig. 2 shows the block diagram of the smoothing technique. The Hatch filter can be expressed as

$$
\bar{X}[k]=\frac{1}{\alpha} X[k]+\left(1-\frac{1}{\alpha}\right) \bar{X}[k-1],
$$

where $\alpha$ is the smoothing time constant in terms of number of samples, $\bar{X}[k]$ is the filtered version of $X=\Psi-\Phi$ i.e. the different between the two estimated measurements, which can be defined as

$$
\begin{aligned}
& \Psi=r+I_{\Psi}+w_{\Psi}, \\
& \Phi=r+I_{\Phi}+w_{\Phi}+\lambda,
\end{aligned}
$$

where $r$ is the distance between the satellite and the receiver and is the same for both delays, $I_{\Psi}$ and $I_{\Phi}$ are the ionosphere delays for both measurements and could be different, $w_{\Psi}$ and $w_{\Phi}$ are the thermal noise and multipath effect and $\lambda$ is the ambiguity term. The smoothed measurement can be written as

$$
\bar{\Psi}[k]=\bar{X}[k]+\Phi[k] .
$$

Substituting (3) and (2) in (4), the smoothed measurement can be written as

$$
\begin{aligned}
& \bar{\Psi}[k]=\left(r[k]+I_{\Phi}[k]+w_{\Phi}[k]+\lambda\right)+ \\
& +\frac{1}{\alpha}\left(r[k]+I_{\Psi}[k]+w_{\Psi}[k]\right) \\
& +\frac{1}{\alpha}\left(-r[k]-I_{\Phi}[k]-w_{\Phi}[k]-\lambda\right)+ \\
& +\left(1-\frac{1}{\alpha}\right)\left(\bar{r}[t-1]+\bar{I}_{\Psi}[t-1]+\bar{w}_{\Psi}[t-1]\right)+ \\
& +\left(1-\frac{1}{\alpha}\right)\left(-\bar{r}[t-1]-\bar{I}_{\Phi}[t-1]-\bar{w}_{\Phi}[t-1]-\lambda\right),
\end{aligned}
$$

where we have used the following definitions

$$
\begin{aligned}
& \bar{I}_{\Psi}[k]=\frac{1}{\alpha} I_{\Psi}[k]+\left(1-\frac{1}{\alpha}\right) \bar{I}_{\Psi}[t-1], \\
& \bar{I}_{\Phi}[k]=\frac{1}{\alpha} I_{\Phi}[k]+\left(1-\frac{1}{\alpha}\right) \bar{I}_{\Phi}[t-1], \\
& \bar{w}_{\Psi}[k]=\frac{1}{\alpha} w_{\Psi}[k]+\left(1-\frac{1}{\alpha}\right) \bar{w}_{\Psi}[t-1], \\
& \bar{w}_{\Phi}[k]=\frac{1}{\alpha} w_{\Phi}[k]+\left(1-\frac{1}{\alpha}\right) \bar{w}_{\Phi}[t-1] .
\end{aligned}
$$

The expression in (5) can be written as

$$
\begin{aligned}
& \bar{\Psi}[k]=r[k]+ \\
& +I_{\Phi}[k]+w_{\Phi}[k]+\bar{I}_{\Psi}[k]-\bar{I}_{\Phi}[k]+\bar{w}_{\Psi}[k]-\bar{w}_{\Phi}[k] .
\end{aligned}
$$


The last two terms are the filtered noise of both measurements. Compare to the sampled noise, these two terms can be neglected. $\bar{I}_{\Psi}[k]$ and $\bar{I}_{\Phi}[k]$ are the filtered or smoothed delays due to the ionosphere.

\section{B. Carrier Smoothing}

The carrier smoothing method is well known in the GNSS community: the carrier measurements are used jointly with the code measurements in order to reduce the code noise. In this case, the generalized variables presented in the previous section can be formulated as $\Psi=\rho$ (code measurements )and $\Phi=\phi$ (phase measurements). The delay introduced by the ionosphere is well documented [9] $I_{\Psi}=-I_{\Phi}=I$. Then, (7) can be written as

$$
\bar{\rho}[k]=r[k]+I[k]+w_{\phi}[k]+2 \bar{I}[k]+\bar{w}_{\rho}[k]-\bar{w}_{\phi}[k] .
$$

The last equation shows the dependence on the previous evolution ionosphere effect. As it is time depending a large integration time can cause an unacceptable bias.

\section{CODE SMOOTHING STRATEGY}

In this section a different smoothing strategy is presented, its schematic is shown in Fig. 3. There are three independent loops: The full BOC and the two BPSK tracking loops. Nevertheless, the phase measurements $\left(\theta_{\text {carrier }}\right)$ from the full BOC can be supplied to the BPSK loops. The code measurements from the two BPSK loops $\left(\tau_{U}\right.$ and $\left.\tau_{L}\right)$ are combined to each other getting the $\tau_{\mathrm{BPSK}}$ measurement. Finally, It is smoothed with the full BOC measurement $\left(\tau_{\mathrm{BOC}}\right)$ obtaining the unambiguous and smoothed $\bar{\tau}$ measurement.

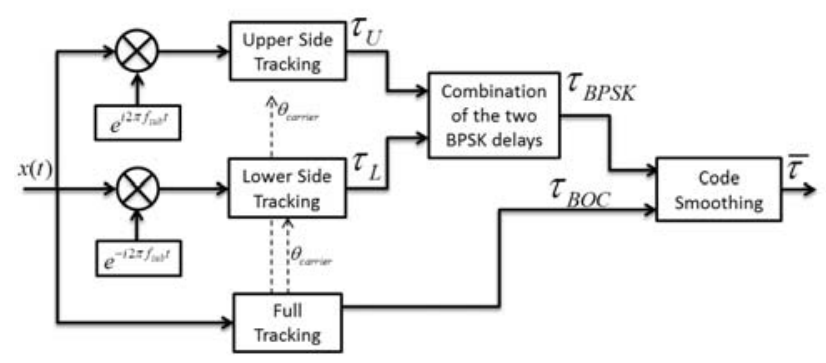

Fig. 3. Code Smoothing schematic

The generalized variables can be reformulated for code smoothing as $\Psi=\tau_{\mathrm{BPSK}}$ and $\Phi=\tau_{\mathrm{BOC}}$. Assuming that both delays are affected in the same way by the ionosphere, i.e. $I_{\Psi}=I_{\Phi}=I$, (7) is written as

$$
\begin{aligned}
& \bar{\tau}[k]= \\
& =r[k]+I[k]+w_{\tau_{\mathrm{BOC}}}[k]+\bar{w}_{\tau_{\mathrm{BPSK}}}[k]-\bar{w}_{\tau_{\mathrm{BOC}}}[k] .
\end{aligned}
$$

The result achieved is very interesting. The measurement does not depend on the past evolution of the ionosphere effect. Therefore a large smoothing time can be done. It has to be taken into account that a change in the ambiguity produces the same effect as a cycle slip. Moreover, if a large smoothing time is set the filtered terms in the equation can be neglected. Then, the measurement can be approximated as

$$
\bar{\tau}[k] \approx r[k]+I[k]+w_{\tau_{\mathrm{BOC}}}[k] .
$$

In order to reduce the the time of convergence, when code smoothing starts, $\alpha$ increases linearly until it reaches its defined value.

\section{IONOSPHERE EFFECT ON BOC SIGNALS}

In the above section it has been assumed that the effect of the ionosphere for both code measurements is the same. The main purpose of this section is to present the ionospheric model and demonstrate that the effect of the ionosphere can be considered equivalent when BPSK-Like and Full BOC techniques are implemented. Besides, a simplified expression of the ionosphere effect for narrowband signals is presented. There are many documents in the literature that describe the ionosphere effect such as [9]. The refraction index for the phase propagation in the ionosphere can be approximated as

$$
n_{p}=1+\frac{c_{2}}{f^{2}}+\frac{c_{3}}{f^{3}}+\frac{c_{4}}{f^{4}}+\ldots,
$$

where all the $c_{x}$ coefficients only depend on the number of electrons, but not on the frequency. $c_{2}$ is estimated as $c_{2}=-40.3 n_{e} \mathrm{~Hz}^{2}$, where $n_{e}$ is the electron density. All the other terms, bigger than $c_{2}\left(c_{3}, c_{4}\right)$, can be neglected for our purposes. The group refractive index can be determined by

$$
n_{g r}=n_{p}+f \frac{d n_{p}}{d f} .
$$

Substituting $c_{2}$ in (11) and (12) and neglecting all the terms bigger than $c_{2}$, both index can be written as

$$
n_{p}=1-\frac{40.3 \mathrm{n}_{e}}{f^{2}}, \quad n_{g r}=1+\frac{40.3 \mathrm{n}_{e}}{f^{2}} .
$$

The phase refractive index is less than the unity, hence the phase velocity is greater than the speed of light in vacuum, i.e. the phase suffers an advance. Nevertheless, the group refractive index is bigger than the unit, therefore the group velocity is less than the light speed in vacuum. Integrating $n_{p}$ and $n_{g r}$ along the signal path, the phase and code measurements are obtained. In [9] the author shows that, after the path integration, the phase and the group delay introduced by the ionosphere can be expressed as

$$
\tau_{p}=-\frac{40.3 \mathrm{TEC}}{f^{2}}, \quad \tau_{g r}=\frac{40.3 \mathrm{TEC}}{f^{2}},
$$

where TEC represents the total electron in units of TECU $=$ $10^{16} \mathrm{e} / \mathrm{m}^{2}$ and $f$ is the frequency of the signal. Both equations have units of meters. For instance, if TEC $=1$ TECU the delay introduced by the ionosphere is 0.1624 meters. Taking into account the effect on the phase and group delay, the ionosphere can be modeled as a filter with response

$$
H(f)=e^{\frac{j 2 \pi 40.3 \mathrm{TEC}}{c f}},
$$

where $c$ is the light speed. Assuming that the two main lobes of the BOC signals are narrow enough, each one of them can be approximated to only one frequency. Then, the ionosphere 
filter can be expressed by the first order of Taylor's series expansion

$$
\begin{aligned}
& \angle H(f) \approx \angle H\left(f_{0}\right)+\left[\frac{d}{d f} \angle H(f)\right]_{f=f_{0}}\left(f-f_{0}\right) \\
& \approx \frac{2 \pi 40.3 T E C}{c f_{0}}+\left[-\frac{2 \pi 40.3 T E C}{c f^{2}}\right]_{f=f_{0}}\left(f-f_{0}\right) \\
& \approx 2\left(\frac{2 \pi 40.3 T E C}{c f_{0}}\right)-\frac{2 \pi f 40.3 T E C}{c f_{0}^{2}} .
\end{aligned}
$$

\section{BPSK-CODE COMBINATION}

The main goal of this section is to analyse whether the delay introduced by the ionosphere when the matched filter is applied is the same as the one achieved by the BPSK-Like technique.

Neglecting the multipath, noise and any other effect, the total distance estimated is the distance between the satellite and the receiver $r$ plus the delay, in meters, introduced by the ionosphere i.e. $40.3 \mathrm{TEC} / f^{2}$ meters. Then, assuming that the bandwidth of the signal is narrow enough, it could be written as

$$
\tau_{\text {total }}=\frac{40.3 \mathrm{TEC}}{f^{2}}+r .
$$

The two measurements obtained with the BPSK-Like technique are affected by the same concentration of electrons and the distance between the satellite and the receiver is the same. Hence, the only variable for all the equations is the centered frequency. The following system of equation holds

$$
\begin{aligned}
\tau_{\mathrm{BOC}} & =\frac{40.3 \mathrm{TEC}}{f_{R F}^{2}}+r, \\
\tau_{U} & =\frac{40.3 \mathrm{TEC}}{f_{U}^{2}}+r, \\
\tau_{L} & =\frac{40.3 \mathrm{TEC}}{f_{L}^{2}}+r .
\end{aligned}
$$

One can realize that adding the two BPSK measurements, the full BOC one is not achieved. In order to get it the following combination must be applied

$$
\begin{aligned}
& \tau_{\mathrm{BPSK}}= \\
& =\frac{f_{U}^{2}}{f_{U}^{2}-f_{L}^{2}}\left(1-\frac{f_{L}^{2}}{f_{R F}^{2}}\right) \tau_{U}-\frac{f_{L}^{2}}{f_{U}^{2}-f_{L}^{2}}\left(1-\frac{f_{U}^{2}}{f_{R F}^{2}}\right) \tau_{L},
\end{aligned}
$$

where:

- $f_{R F}$ is the carrier frequency

- $f_{U}$ is the carrier frequency plus the sub-carrier frequency

- $f_{L}$ is the carrier frequency minus the sub-carrier frequency

- $\tau_{U}$ is the measurement estimated with the upper BPSK

- $\tau_{L}$ is the measurement estimated with the lower BPSK

In order to corroborate the above combination several simulations have been carried out. Non coherent discriminator has been applied with the same Early-Late spacing. Fig. 4 shows the error of the ionospheric delay estimation obtained using the two BPSK and the full BOC for different values of TEC at E1 band. The green color is the difference between both errors. It should be noted that, the distance introduced by the ionosphere, for instance, for TEC $=150$ is around

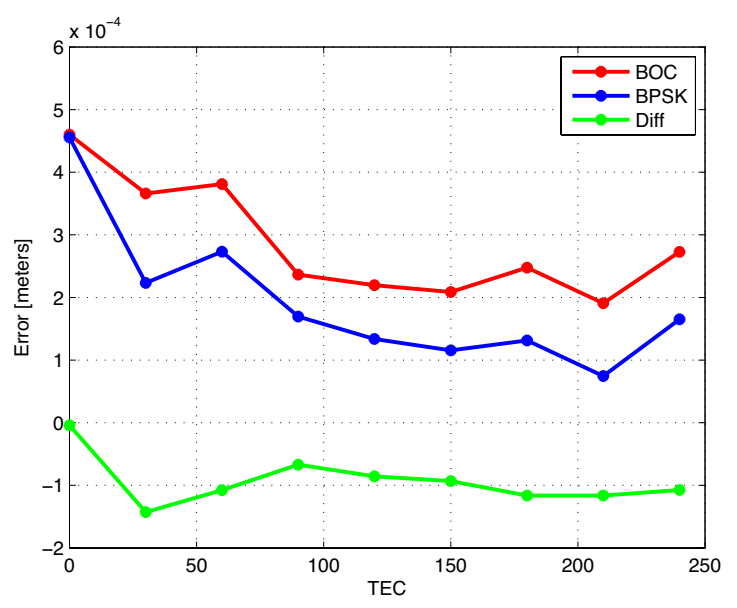

Fig. 4. Ionosphere Delay Estimation Error for BOC and BPSK-Like

24.3559 meters and the error in the estimation is around 0.01 centimeter. The simulation has been done using the ionosphere mode shown in (15). Since the multipath and even the thermal noise cause larger error in the time-delay measurements, this difference can be neglected.

\section{RESULTS}

In this section the code smoothing results are presented. The common receiver parameters are:

- $\operatorname{BOC} \cos (15,2.5)$

- CodePeriod $=0.01$ seconds

- $f s=123 \mathrm{Msps}$

- $\mathrm{BWPLL}=10 \mathrm{~Hz}$

- BWDLL $_{\mathrm{BPSK}}=1 \mathrm{~Hz}$

- $\mathrm{BWDLL}_{\mathrm{BOC}}=1 \mathrm{~Hz}$

- EarlyLateSpacingBPSK $=0.08$ chips

- EarlyLateSpacingBOC $=0.041$ chips

Before showing the properties against noise or filtering, the ambiguity mitigation is demonstrated. Fig. 5 shows the code smoothing and the full BOC measurements when the initial error is 12 meters, i.e. the tracking full BOC loop starts at a side lobe and it remains there. The code smoothing goes to the main lobe. The smoothing time has been set to 2 second.

Since the smoothing time constant is increasing during the transition, the time to reach the final value does not depend, or almost not, on the time constant. Fig. 6 shows the transition epoch for different $\alpha$ values. For all the simulations, the units of $\alpha$ are in epochs, i.e. 0.01 seconds. As it can be seen, the convergence time is almost the same. However, as the time constant increases, the fluctuations decrease. It should be noted that the transition is achieved without any jump. The transition time depends, among other factors, on the DLL parameters, the early late spacing and the discriminator, such as the bandwidth of the filter.

In the Section III the capability to achieve the same performance as the full BOC tracking, in terms of standard 


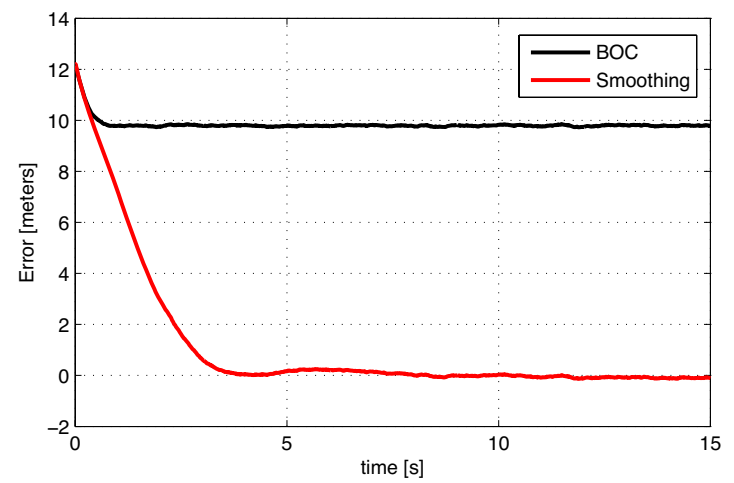

Fig. 5. Code Smoothing Ambiguity

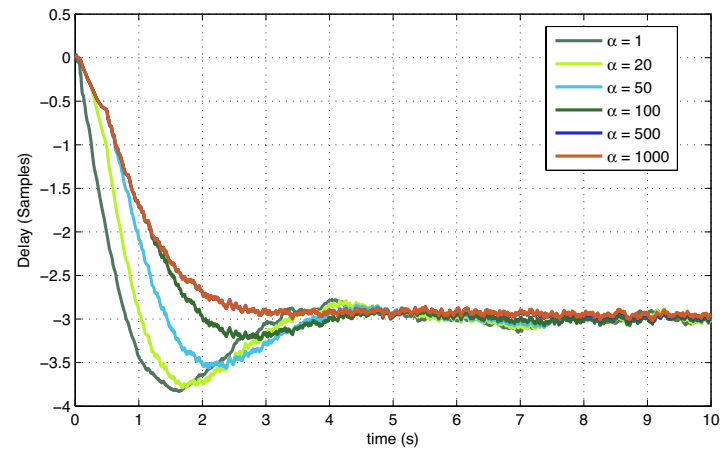

Fig. 6. Code Smoothing Transition Time for different values of $\alpha$

deviation, has been demonstrated for high values of time smoothing constant. Fig. 7 shows the estimation of the code smoothing strategy versus full BOC for $C / N o=33 \mathrm{dBHz}$ and $C / N_{O}=40 \mathrm{dBHz}$. It should be noted that for time smoothing constant of 1000 epochs, i.e. 10 seconds, the behavior is almost the same as the Full BOC tracking. Table I presents the standard deviation for different time constants. The bigger the time is, the smaller the variance is.

When the signal passes through a filter with non-linear phase response, i.e. non-constant group delay, a shift in the

TABLE I

STANDARD DEVIATION OF CODE SMOOTHING VS FULL BOC.

\begin{tabular}{ccc}
\hline & \multicolumn{2}{c}{$\sigma$ [meters] } \\
\cline { 2 - 3 }$\alpha$ [epoch] & $C / N_{0}=40 d B H z$ & $C / N_{0}=33 d B H z$ \\
\hline 50 & 0.65 & 0.28 \\
100 & 0.51 & 0.23 \\
1000 & 0.25 & 0.14 \\
5000 & 0.20 & 0.12 \\
\hline BOC & 0.20 & 0.12 \\
\hline
\end{tabular}
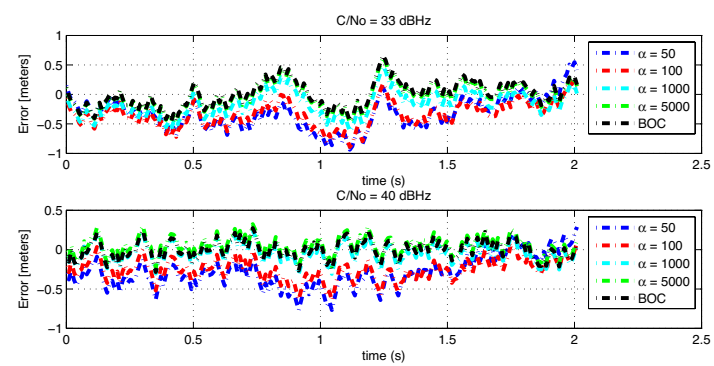

Fig. 7. Code Smoothing vs. full BOC for different values of $\alpha$

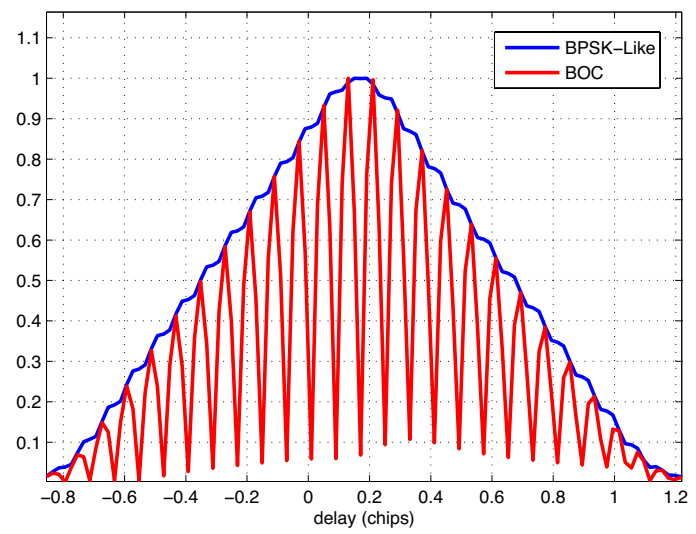

Fig. 8. Impact of a Butterworth filter on the BOC correlation and its envelope

correlation function relative to its envelope is produced. This shift may bring the sub-carrier tracking point close to a side lobe. In a nutshell, the maximum of the sub-carrier correlation function is separated from the maximum of the envelope. The advantage of code smoothing technique is that it follows the mean value of the envelope and the variance of the full BOC, or close. Since the error is the same for all the satellites it has no affect when the PVT is performed. Fig. 8 shows the full BOC correlation and the one achieved with the BPSK envelope when the signal has been filtered with a Butterworth filter (non-linear phase response) of order 6 and $42 \mathrm{MHz}$ of bandwidth.

Other techniques, as the DE, need to know the bias between the maximum of the full BOC and the BPSK envelope and correct it before the codes combination. Fig. 9 shows the impact of a Butterworth filter on the smoothing strategy. The smoothing time is 1000 epochs. The bias between the BPSK and the full BOC delays is 2.8 meters. The smoothed delay follows the mean value of BPSK and uses the full BOC measurements for the punctual updates, hence, it does not suffer jumps. However, the DE follows the full BOC and compares these measurements with the DLL measurements. It jumps if the difference between them is bigger than half subcarrier cycle (4.887 meters). Since there is a constant bias, the range decreases, and then, some undesired jumps happen. 


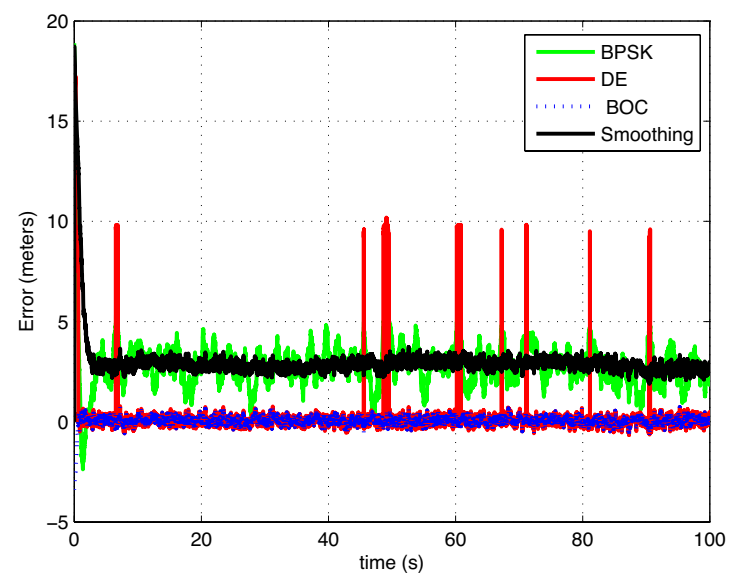

Fig. 9. Code Smoothing with Non Linear Group Delay filter

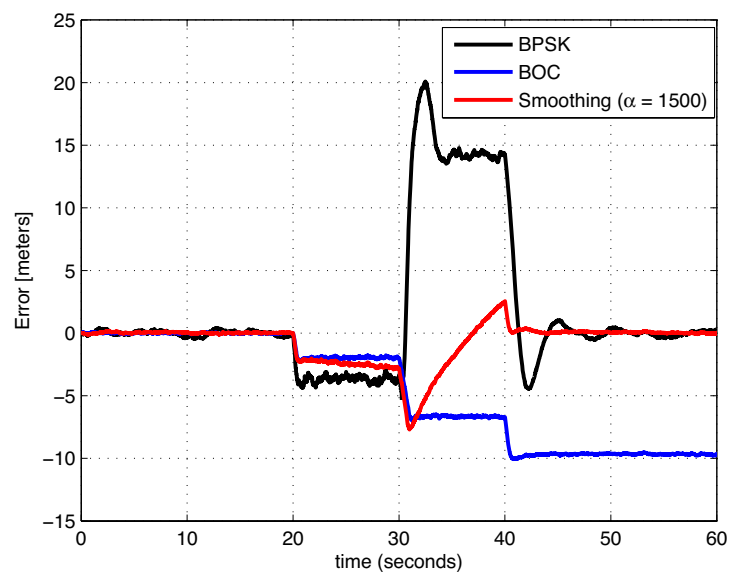

Fig. 10. Code Smoothing with multipath scenario

Since the smoothing strategy is a filter itself, it is not straightforward to evaluate its behavior against multipath. Due the memory of the technique the evolution of the multipath must be taken into account. Nevertheless, Fig. 10 shows the smoothing measurements versus the BOC and BPSK ones. The scenario simulated is really interesting because it causes a false lock in the BOC loop. A strong multipath, only $2 \mathrm{~dB}$ below the line-of-sight (LOS), appears at 20 seconds, after 10 seconds its power increases above the LOS. At instant 40 the multipath disappears, only the LOS remains. The smoothing strategy is able to get back to the main one. The BOC loop is locked in a side lobe and it is not able to get back to the main one.

\section{CONCLUSIONS}

In this paper a new unambiguous tracking method based on the smoothing strategy has been presented. Besides, a judicious combination of the two delays yielded from the dual side tracking is performed. This combination is then smoothed with the match filter. It is noteworthy that the smoothing strategy can be applied with only one side tracking measurement, any other unambiguous technique, or even the two delays used in the DE.

If there is a constant bias between the unambiguous and the ambiguous measurements, the smoothed strategy follows the mean value of the unambiguous one. For instance, when the signal has passed through a filter with non-continuous group delay. Since the bias is the same for all the satellites it does not affect the PVT solution.

The method presented in this paper shows that using a large smoothing time, the tracking variance is very similar to the one achieved with the ambiguous measurements. The main advantage, over other combination methods, is that this technique never jumps. Moreover, since the integration time can be very large, the effect on the smoothed measurement, due to the multipath and the noise, is the same, or close, as the one obtained with full BOC.

After the results presented in this paper, the future lines of research are the behavior with a real channel, i.e. ionospheric effect, multipath effect and fading. It should be noted that the technique is a filter itself, the multipath envelope is not enough for characterizing the technique, since the error depends on the multipath delay but also on its duration. Moreover, the smoothed measurement has memory due to the filtering. Therefore, the evolution of the channel must be also taken into account.

\section{ACKNOWLEDGEMENTS}

This work was supported in part by the Spanish Ministry of Economy and Competitiveness projects TEC 2011-28219 and EIC-ESA-2011-0080.

\section{REFERENCES}

[1] N. Martin, V. Leblond, G. Guillotel, and V. Heiries, "BOC (x, y) signal acquisition techniques and performances," in Proc. of ION GPS/GNSS, 2003, pp. 188-198.

[2] P. M. Fishman and J. W. Betz, "Predicting performance of direct acquisition for the M-code signal," in Proc. of ION NTM, 2000, pp. 574-582.

[3] A. Burian, E. Lohan, and M. Renfors, "BPSK-like methods for hybridsearch acquisition of galileo signals," IEEE International Conference on Communications ICC '06, vol. 11, pp. 5211-5216, 2006.

[4] V. Heiries, D. Roviras, L. Ries, and V. Calmettes, "Analysis of non ambiguous BOC signal acquisition performance Acquisition," Proc. of ION ITM, pp. 2611 - 2622, 2004.

[5] M. Hodgart, P. Blunt, and M. Unwin, "The optimal dual estimate solution for robust tracking of Binary Offset Carrier (BOC) modulation," in Proc. of ION GNSS, 2007, pp. 1017-1027.

[6] C. Palestini, "Synchronization and detection techniques for navigation and communication systems," Ph.D. dissertation, Univ. of Bologna, 2010.

[7] C. Gunther and P. Henkel, "Reduced-noise ionosphere-free carrier smoothed code," IEEE Transactions on Aerospace and Electronic Systems, vol. 46, no. 1, pp. 323-334, 2010.

[8] S. Sen and J. Rife, "Nonlinear filter for ionosphere divergence error reduction in LAAS," IEEE Transactions on Aerospace and Electronic Systems, vol. 48, no. 2, pp. 981-990, 2012.

[9] E. D. Kaplan and C. Hegarty, Understanding GPS: principles and applications. Artech House Publishers, 2005. 